\title{
Revascularización coronaria híbrida: un punto de encuentro entre Cardiólogos Intervencionistas y Cirujanos Cardíacos
}

\author{
René Hameau ${ }^{1,2}$, Pablo Sepúlveda ${ }^{1}$, Osvaldo Pérez ${ }^{2}$, Joaquín Gundelach ${ }^{3}$, Maurice Cortés $^{1,4}$, Gonzalo Martínez ${ }^{1}$, Alberto Fuensalida ${ }^{1}$, \\ Alejandro Martínez ${ }^{1^{*}}$, Ricardo Zalaquett $5^{*}$. \\ 1 Centro de Terapia Endovascular, Hospital Clínico Pontificia Universidad Católica, Santiago, Chile. \\ 2 Unidad de Medicina Cardiovascular Integrada, Hospital Las Higueras, Talcahuano, Chile. \\ 3 Unidad de Cirugía Cardíaca, Hospital Las Higueras, Talcahuano, Chile. \\ 4 Unidad de Electrofisiología, Instituto Nacional del Tórax, Santiago, Chile. \\ 5 Unidad de Cirugía Cardiovascular, Hospital Clínico Pontificia Universidad Católica, Santiago, Chile. \\ *Ambos autores contribuyeron en igual proporción a la redacción del manuscrito.
}

La revascularización coronaria híbrida busca combinar el beneficio de las técnicas quirúrgicas y percutáneas para un manejo óptimo de pacientes seleccionados con enfermedad coronaria obstructiva multivaso. Esto permite asociar el beneficio del puente de arteria mamaria interna izquierda a la arteria descendente anterior (ADA) y combinarlo con el implante de stents en lesiones no-ADA. El objetivo de este tra- bajo es hacer una revisión de la literatura disponible con énfasis en sus resultados clínicos comparados con la estrategia convencional.

Palabras clave: Revascularización híbrida coronaria, enfermedad de arteria coronaria, cirugía de bypass de arteria coronaria, intervención coronaria percutánea.

\section{Hybrid coronary revascularization: A meeting point between interventional Cardiologists and Cardiac Surgeons}

Hybrid coronary revascularization seeks to combine the benefit of surgical and percutaneous techniques for optimal management of selected patients with multivessel coronary artery disease. This allows combining the benefit of the left internal mammary artery bypass to the anterior descending artery (LAD) and stent deployment in non-LAD lesions. The objective of this manuscript is to review the available literature with emphasis on its clinical results compared to the conventional strategy.

Keywords: Hybrid coronary revascularization; coronary artery disease; coronary artery bypass graft surgery; percutaneous coronary intervention

\section{Correspondencia:}

Dr. Alejandro Martínez S.

amartine@med.puc.cl

Dr. Ricardo Zalaquett S.

rzalaque@med.puc.cl 


\section{Introducción:}

La cirugía de revascularización miocárdica (CRM) y la angioplastía coronaria (PCI, del inglés, "percutaneous coronary intervention") son las principales estrategias actuales para el tratamiento de pacientes con enfermedad coronaria multivaso, siendo la CRM considerada el "gold standar" en muchos de los casos debido al beneficio del injerto de arteria mamaria izquierda en territorio de la arteria descendente anterior. A pesar de que el estudio SYNTAX ${ }^{1}$ ayudó a establecer ciertas categorías anatómicas que orientan hacia la mejor estrategia de tratamiento, existen otros elementos clínicos a considerar en la toma de decisiones asi como un potencial de mejoría en los resultados de ambas técnicas.

Es en este escenario que surge el concepto de Revascularización Coronaria Híbrida (RCH), referido al uso de técnicas mínimamente invasivas para realizar un bypass de arteria mamaria interna izquierda (LIMA, del inglés "left internal mammary artery") a la arteria descendente anterior (ADA) más una PCI a las lesiones remanentes en la arteria circunfleja (ACx) o la arteria coronaria derecha (ACD), en un mismo procedimiento, o dentro de un período de 60 días.

Descrita inicialmente por Angelini et $\mathrm{al}^{2}$ en 1996 , la $\mathrm{RCH}$ representaría una alternativa a la CRM tradicional por esternotomía o a la revascularización completa mediante una PCI multivaso. El planteamiento inicial es que esta modalidad combinaría "lo mejor de ambos mundos": por un lado, los beneficios de LIMA-ADA en términos de permeabilidad a largo plazo, sobrevida libre de eventos ${ }^{3}$, alivio de la angina y, por otro, los beneficios de la terapia percutánea con stents de última generación que, en comparación a los puentes venosos, tiene menor tasa de eventos o reestenosis a largo plazo, así como el hecho de ser menos invasiva y con menores tiempos de recuperación. El objetivo de este artículo es hacer una revisión actualizada de la RCH, incluyendo sus fundamentos teóricos, indicaciones, especificaciones técnicas y resultados clínicos.

\section{Fundamento teórico}

En pacientes con enfermedad multivaso, especialmente aquellos con Diabetes Mellitus tipo 2, que presentan lesiones significativas en la arteria coronaria izquierda, la CRM utilizando LIMA ha demostrado beneficios sustanciales en mortalidad en comparación con PCI o manejo médico. En el seguimiento a largo plazo del estudio BARI $^{4}$, aquellos pacientes diabéticos aleatorizados a CRM con al menos un puente arterial $(n=145)$ tuvieron una sobrevida a 10 años del 64,3\%, en comparación a $39,4 \%$ en aquellos que recibieron CRM sólo con puentes aorto-coronarios (PAC) venosos $(n=33)$ y $45,5 \%$ en los randomizados a $\mathrm{PCI}(\mathrm{N}=63)$.

Los principales beneficios del uso de la LIMA se basan en su alta permeabilidad a largo plazo, llegando a tasas $>90 \%$ a 10 años $^{5}$. Por otro lado, se ha descrito un efecto protector que limita la progresión de la enfermedad sobre el resto del árbol coronario nativo ${ }^{6}$. En contraparte, los resultados del uso de PAC venosos tienden a ser inferiores con tasas de reestenosis que pueden alcanzar entre $10-20 \%$ al año ${ }^{7}$ y hasta $40-50 \%$ a 10 años $^{8}$. Sin embargo, otras series actuales muestran una mejoría al respecto con permeabilidades de hasta $86,4 \%$ a 5 años 9 y $74,3 \%$ a 10 años ${ }^{10}$.

Por otro lado, los nuevos stents medicados de segunda o tercera generación han mostrado mejorías significativas respecto de aquellos de primera generación. En una publicación reciente, Ki et al. ${ }^{11}$ analizaron los datos del registro multicéntrico Grand-DES que incluyó 13.097 pacientes tratados con stent de segunda generación. En el seguimiento a 3 años, se reportaron tasas de trombosis del stent del $0,6 \%$, revascularización de la lesión culpable de $3,2 \%$ y fallas del tratamiento de la lesión culpable (combinado de muerte cardiovascular, infarto agudo al miocardio o revascularización de la lesión culpable) del 6,6\%.

Yano et al. ${ }^{12}$ reporta resultados similares con los stents de última generación con tasas de revascularización de la lesión culpable del 4,6\%, trombosis del stent $1,2 \%$ e infarto agudo al miocardio de $1,4 \%$ a 5 años.

En suma, existe evidencia de que la LIMA - ADA es la mejor opción para la revascularización de la pared anterior en términos de sobrevida y permeabilidad a largo plazo pero que los stents medicados actuales sobre lesiones no-ADA serían iguales o superiores al uso de puentes venosos respecto de eventos clínicos incluyendo la reestenosis. Este marco teórico explica la racionalidad de combinar ambas estrategias para lograr un beneficio clínico neto con la RCH.

\section{Selección de pacientes y especificaciones técnicas}

Un requerimiento esencial de esta estrategia es que exige una estrecha comunicación entre el equipo Cardio-quirúrgico y el de Cardiología Intervencional para la selección adecuada de pacientes. La Figura 1 muestra un algoritmo de toma de decisiones terapéuticas.

En 2016, Rosenblum et al ${ }^{13}$ analizaron el registro de 306 pacientes consecutivos sometidos a $\mathrm{RCH}$ y que fueron comparados con 8254 pacientes que recibieron CRM con 
Figura 1: Algoritmo de toma de decisiones terapéuticas.

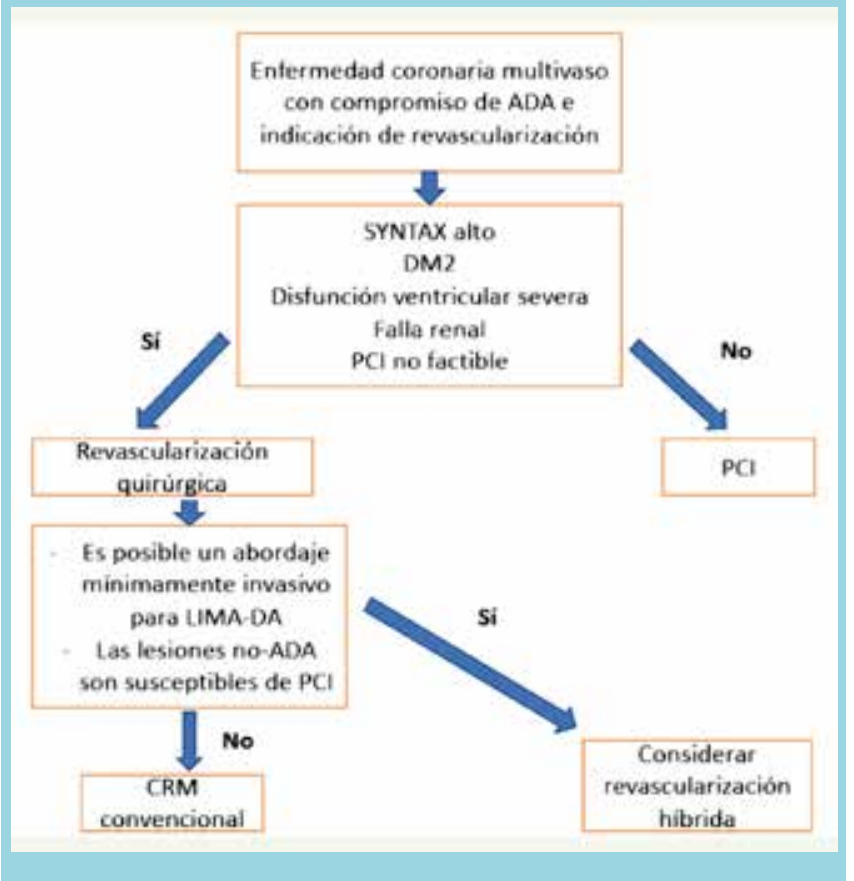

LIMA $(7.381 ; 89,4 \%)$ o LIMA+RIMA $(873 ; 10,6 \%)$. Los principales predictores de $\mathrm{RCH}$ fueron: edad avanzada, bajo IMC, historia de PCI previa y la presencia de enfermedad sólo de 2 vasos. Por otro lado, las contraindicacio- nes relativas más frecuentes fueron: obesidad mórbida, enfermedad pulmonar severa e inestabilidad hemodinámica. Cabe destacar que este estudio no mostró diferencias en la tasa de eventos adversos cardiovasculares mayores (MACE) (RCH 3,3\% vs CRM 2,2\%, p NS) pero sí una reducción significativa en la necesidad de transfusiones $(\mathrm{p}<0,001)$, tiempo en ventilación mecánica y estadía hosptalaria.

A pesar de no existir protocolos técnicos, existe acuerdo entre los expertos respecto de algunas consideraciones para realizar esta estrategia. En el ámbito quirúrgico, la principal diferencia está constituida por el uso de la técnica de bypass coronario mínimamente invasivo ("Minimally invasive direct coronary artery bypass (MIDCAB)") (Figura 2). Básicamente, consiste en que la disección de la LIMA se realiza a través de una mini-toracotomía (5-6 cm) anterior izquierda en $5^{\circ}$ espacio intercostal y la anastomosis se realiza sin circulacion extracorporea de forma estándar, por videotoracoscopía o por técnica robótica ${ }^{14}$. Entre los principales beneficios se menciona: 1) Evita una esternotomía, no requiere clampeo aórtico y provoca menor dolor postoperatorio. 2) Reducción de la morbilidad del procedimiento (eventos adversos neurológicos, sangrado, infección, complicaciones pulmonares) y, probablemente, mejoría en desenlaces clínicos (menor estadía hospitalaria) ${ }^{15}$. Dentro de sus limitaciones des-

Figura 2: Técnica de bypass coronario mínimamente invasivo.

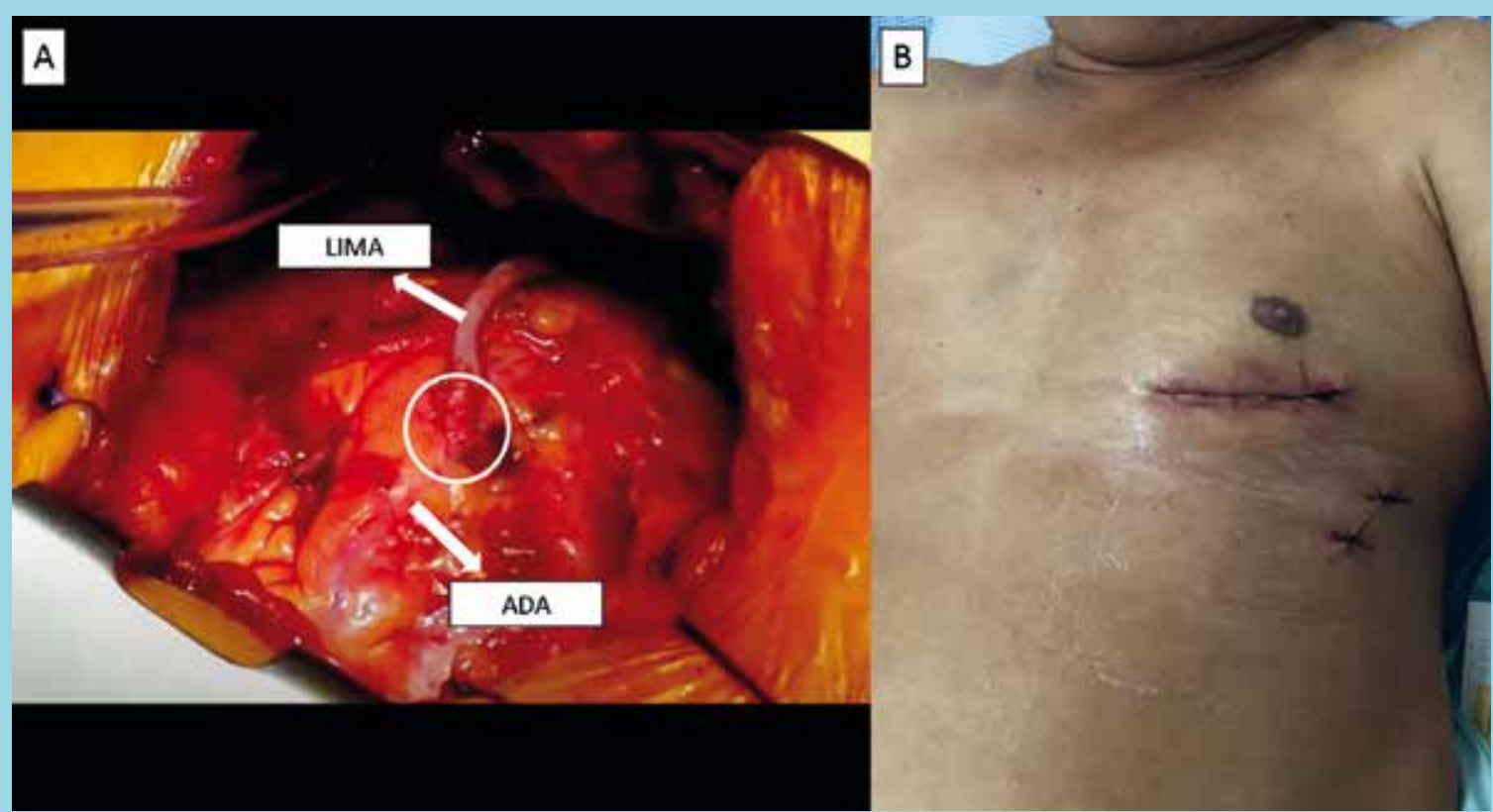

A) Imagen intraoperatoria que muestra campo quirúrgico por mini-toracotomía con anastomosis (círculo) de la arteria mamaria interna izquierda (LIMA) a la arteria descendente anterior (ADA). B) Cicatriz de mini-toracotomía. 
Tabla 1:

Esquema comparativo de las distintas secuencias para realizar la revascularización híbrida (Adaptado de Panoulas et al ${ }^{27}$ ).

\begin{tabular}{|c|c|c|}
\hline 1 Etapa & \multicolumn{2}{|c|}{2 Etapas } \\
\hline $\begin{array}{l}\text { MIDCAB seguido de } \mathrm{PCl} \text { en el mismo } \\
\text { procedimiento }\end{array}$ & MIDCAB 1ro $\rightarrow$ PCI 2do & $\mathrm{PCl} 1 \mathrm{ro} \rightarrow \mathrm{MIDCAB} 2 \mathrm{do}$ \\
\hline \multicolumn{3}{|c|}{ Ventajas } \\
\hline $\begin{array}{l}\text { - Permite evaluación angiográfica del bypass } \\
\text { UMA-DA. } \\
\text { - En caso de fallar la angioplastia, pudiera } \\
\text { reconvertirse a CRM convencional. } \\
\text { - Costo-efectivo al reducir la estadia } \\
\text { hospitalaria. } \\
\text { Mayor satisfacción usuaria al resolver todo en } \\
\text { un único tiempo. }\end{array}$ & $\begin{array}{l}\text { - Permite evaluación angiográfica del bypass } \\
\text { LMA-DA. } \\
\text { - Doble antiagregación completa post MIDCAB, } \\
\text { sin riesgo de sangrado perioperatorio. } \\
\text { - Pared anterior protegida por UMA, } \\
\text { disminuyendo riesgo de la PCI. }\end{array}$ & $\begin{array}{l}\text { - Permite evaluación del calibre de la UMA } \\
\text { previo la cirugia. } \\
\text { - Menor riesgo de isquemia durante MIDCAB } \\
\text { en un corazón parcialmente revascularizado. } \\
\text { - Útil en el contexto de IAM donde la lesión } \\
\text { culpable no es la ADA. }\end{array}$ \\
\hline \multicolumn{3}{|c|}{ Desventajas } \\
\hline $\begin{array}{l}\text { - Sólo posible en centros experimentados y con } \\
\text { pabellones hibridos. } \\
\text { - Respuesta inflamatoria por la MIDCAB puede } \\
\text { aumentar riesgo de trombosis del stent. } \\
\text { - Terapia antiplaquetaria dual aumenta riesgo } \\
\text { de sangrado. } \\
\text { - Mayor riesgo de falla renal dada injuria } \\
\text { asociada a la cirugia + uso de medio de } \\
\text { contraste. }\end{array}$ & $\begin{array}{l}\text { - Riesgo de isquemia en los territorios no-ADA } \\
\text { durante la cirugia (Poco probable en pacientes } \\
\text { estables). } \\
\text { - En caso de fallar la angioplastia, existe un } \\
\text { mayor riesgo de la reintervención quirúrgica. }\end{array}$ & $\begin{array}{l}\text { - No hay control angiográfico del bypass LIMA- } \\
\text { DA. } \\
\text { - Mayor riesgo de trombosis del stent durante } \\
\text { la cirugia. } \\
\text { - Mayor riesgo de sangrado perioperatorio por } \\
\text { terapia antiagregante dual. } \\
\text { - Riesgo de presentar eventos en el territorio } \\
\text { de la ADA mientras espera cirugia. }\end{array}$ \\
\hline
\end{tabular}

taca la contraindicación de su uso en pacientes con patología pulmonar severa (Ventilación mono-pulmonar). Una de las primeras experiencias nacionales con técnica mínimamente invasiva fue reportada por Zalaquett et al. en $1999^{23}$.

Respecto de la terapia percutánea, los únicos requisitos son que las lesiones en vasos no-ADA sean susceptibles de una angioplastía y que deben ser realizados con stent medicados de 2da o 3ra generación.

En cuanto al orden en que se realizan las intervenciones, cada estrategia posee sus ventajas y desventajas particulares (Tabla 1), si bien la opción más frecuente hoy en día es realizar primero la MICAB y luego realizar en diferido la angioplastía coronaria.

Respecto de la terapia antiagregante plaquetaria, se requiere balancear el riesgo de sangrado perioperatorio con el de trombosis del stent. En la mayoría de las series con "MIDCAB", esta se realizó sólo con Aspirina y se agregó un $2^{\circ}$ antiplaquetario luego de 4 hrs, una vez descartadas complicaciones hemorrágicas ${ }^{16}$. En los casos de "PCI- primero", la intervención se realizó con antiagregación dual y esta se mantuvo ininterrumpida durante la MIDCAB ${ }^{17}$.

\section{Evidencia clínica}

En 2014 , Gasior et al ${ }^{18}$ publica un estudio de 200 pacientes con lesión en $\mathrm{ADA}>70 \%$ + una o más lesiones en otros territorios, aleatorizados a una CRM convencional $(n=102)$ o RCH $(n=98)$. El score de SYNTAX promedio en ambos grupos era de $22,8 \pm 5,3$ y 23,4 \pm 6,3 , respectivamente, y el tiempo promedio desde la MIDCAB a la angioplastía fue de $21 \pm 5,7$ hrs. En el seguimiento a 12 meses, no hubo diferencias significativas en la tasa de eventos adversos cardiovasculares mayores (MACE) (CRM 7,8\% vs RCH 10.2\%, (p log-rank 0,54). En el seguimiento a 5 años, la mortalidad por todas las causas fue similar en los 2 grupos $(6,4 \% \mathrm{RCH}$ vs. 9,2\% CRM; $=0,69)$. Las tasas de infarto de miocardio $(4,3 \%$ vs. $7,2 \% ; \mathrm{p}=0,30)$, revascularización repetida $(37,2 \%$ vs. $45.4 \% ; \mathrm{p}=0,38)$, accidente cerebrovascular $(2,1 \%$ vs. $4,1 \% ; \mathrm{p}=0,35)$ y eventos adversos cardíacos y cerebrovasculares adversos $(45,2 \%$ vs $53,4 \% ; \mathrm{p}=0.39)$ 
también fueron similares en los 2 grupos.

La selección de los pacientes parece ser un elemento crucial en los resultados. En 2013, Shet et al ${ }^{19}$, publica una cohorte de 141 pacientes consecutivos sometidos a $\mathrm{RCH}$ en 1 tiempo y se comparó con un grupo de pacientes CRM pareados por score de propensión (propensity score). En aquellos con SYNTAX > 30 puntos, no hubo diferencias en la tasa de MACE de ambos grupos ( $p$ 0,362); destacando también que en aquellos con EuroSCORE > 6, la RCH presentó menos eventos que la CRM $(p=0,030)$. Estos datos subrayan la importancia de la selección de pacientes para $\mathrm{RCH}$ y apoyan la hipótesis que pacientes de mayor riesgo pudieran beneficiarse de una técnica híbrida.

El estudio HRVES ${ }^{20}$ evaluó a 155 pacientes y los aleatorizó 1:1:1 a tratamiento con CRM convencional con LIMA-ADA más puentes venosos $(n=50), \mathrm{RCH}$ con MIDCAB LIMA-DA más angioplastía a vasos remanentes ( $n=52)$ o PCI multivaso con stents liberadores de Everolimus $(n=53)$. El objetivo primario fue la presencia de isquemia residual medido por Tomografía por emisión de positrones (SPECT). A 12 meses, no hubo diferencias significativas entre los 3 grupos $(p<0,45)$. Tampoco hubo diferencias en las tasas de MACE (12\% vs $13,4 \%$ vs $13,2 \%$, respectivamente; $\mathrm{p}<0,83)$ ni infarto agudo al miocardio ( $8 \%$ vs $5,8 \%$ vs $7,5 \%$, respectivamente, $\mathrm{p}<0,66)$. Las tasas de sangrado BARC 3-4 fueron $20 \%$ vs $9,6 \%$ vs $0 \%$ ( $p<0,001)$. Cabe destacar que el control angiográfico a los 12 meses mostró diferencias en las tasas de estenosis significativas del bypass o reestenosis intrastent (CRM 20,4\% vs MIDCAB 8,2\% vs PCI 5,9\%, p<0,05), lo cual pudiera afectar los resultados a largo plazo.

A nivel de recomendaciones internacionales, la guía de revascularización miocárdica de la Sociedad Europea de Cardiología (ESC) entrega una recomendación IIb para el uso de RCH en centros experimentados, destacando que pudiera ser una alternativa atractiva frente a la esternotomía dada su marcada disminución en la estadía hospitalaria, dolor postoperatorio y mejoría más precoz de la calidad de vida. ${ }^{21}$

Un reciente metaanálisis, analizó 9 estudios que incluyeron cerca de 2442 pacientes y comparó la CRM convencional vs RCH. La estrategia híbrida mostró reducciones significativas en las tasas de transfusiones por sangrado postoperatorio (OR 0,43; IC95\% 0,27-0,68), infección (OR 0,19; IC95\% 0,04-0,98) y estadía hospitalaria (6,0 días para RCH vs 7,8 días para CRM).
No hubo diferencias en términos de MACE, infarto agudo al miocardio, accidente cerebrovascular isquémico (ACV) ni mortalidad. Sí hubo una asociación entre $\mathrm{RCH}$ y la necesidad de revascularización del vaso culpable (OR 3,1; IC95\% 1,39 - 6,90).

Finalmente, otro metaanálisis de Sayed Ahmad 22 que incluyó a 9112 pacientes (1.484 en el grupo RCH vs 7.628 en el grupo CRM) mostró que a 30 días no hubo diferencias en mortalidad (OR1,21; IC95\% 0,55-2,66, p 0,64), infarto agudo al miocardio ni desarrollo de fibrilación auricular. La estrategia híbrida tuvo menores requerimientos de transfusiones y estadía en unidad crítica, así como una disminución significativa en la tasa de ACV a 20 meses (OR 0,25; IC95\% 0,07-0,87).

Desde su descripción hace 20 años, la RCH ha sido objeto de numerosos estudios y revisiones. Sin embargo, su tasa de incorporación a la práctica actual es muy baja. A modo de ejemplo, entre julio 2011 y marzo 2013, la base de datos de la Society of Thoracic Surgeons (STS) mostró que la RCH representó sólo el $0.48 \%$ (n= 950) del total $(n=198.622)$ de pacientes sometidos a $\mathrm{CRM}^{24}$. En 2019, un registro del National Cardiovascular Data ${ }^{25}$ encontró que esta técnica fue utilizada sólo en un $0,2 \%$ de los pacientes con enfermedad multivaso. Las principales razones para esto se basan en su complejidad técnica, asi como dificultades en cuanto a disponibilidad de recursos y coordinación logística de 2 procedimientos que requieren protocolos de manejo diferentes. También es relevante considerar que la técnica quirúrgica sin circulación extracorpórea (CEC) no ha demostrado ser superior a la CRM con CEC en términos de eventos cardiovasculares mayores tal como se reportó en el estudio CORONARY ${ }^{26}$. Finalmente, aún falta validación de estudios aleatorizados multicéntricos de mayor volumen para sustentar una mayor difusión del tratamiento híbrido.

\section{Conclusión:}

En conclusión, aunque la evidencia actual sugiere que la $\mathrm{RCH}$ es una técnica segura en un grupo seleccionado de pacientes con enfermedad multivaso, aún no ha demostrado ser superior a la estrategia quirúrgica convencional en términos de eventos cardiovasculares mayores. Por lo tanto, pese a sus fundamentos teóricos, faltarían pruebas que la justifiquen en una alta proporción de los pacientes. Sin embargo, en casos seleccionados por su mayor riesgo quirúrgico, parece ser una opción razonable. 


\section{References:}

1. SERRUYS P, MORICE M, KAPPETEIN A, COLOMBO A, HOLMES D, MACK M et al. Percutaneous coronary intervention versus coronary-artery bypass grafting for severe coronary artery disease. N Engl J Med. 2009;360(1):961-972.

2. ANGELINI G, WILDE P, SALEMO T BG, A C. Integrated left small thoracotomy and angioplasty for multivessel coronary artery revascularisation [2]. Lancet. 1996;347(9003):757-758.

3. Loop F, Lytle B, Cosgrove D, Stewart R, Goormastic M, Williams $\mathrm{G}$ et al. Influence of the Internal-Mammary-Artery Graft on 10-Year Survival and Other Cardiac Events. N Engl J Med. 1986;314(1):1-6.

4. BARI 2D GROUP. The Final 10-Year Follow-Up Results From the BARI Randomized Trial. J Am Coll Cardiol. 2007;49(15):1600-1606.

5. BERGER A, MACCARTHY P, SIEBERT U, CARLIER S, WIJNS W, HEYNDRICKX G et al. Long-term patency of internal mammary artery bypass grafts: Relationship with preoperative severity of the native coronary artery stenosis. Circulation. 2004;110(11):36-40.

6. OTSUKA F, YAHAGI K, SAKAKURA K VR. Why is the mammary artery so special and what protects it from atherosclerosis? Ann Cardiothorac Surg. 2013;2(4):519-526.

7. ALEXANDER J, HAFLEY G, HARRINGTON R, PETERSON E, FERGUSON T, BRUCE L et al. Efficacy and safety of edifoligide, an E2F transcription factor decoy, for prevention of vein graft failure following coronary artery bypass graft surgery: PREVENT IV: A randomized controlled trial. J Am Med Assoc. 2005;294(19):2446-2454.

8. CALISKAN E, RAMOS DE SOUZA D, BÖNING A, LIAKOPOULOS O, CHOI Y, PEPPER J et al. Saphenous vein grafts in contemporary coronary artery bypass graft surgery. Nat Rev Cardiol. 2020;17(3):155-169.

9. PETER C, WEBB C, CHONG C MN. Radial artery versus saphenous vein patency randomized trial: Five-year angiographic follow-up. Circulation. 2008;117(22):2859-2864.

10. TINICA G, CHISTOL R, ENACHE M, CONSTANTIN M, CIOCOIU M FC. Long-term graft patency after coronary artery bypass grafting: Effects of morphological and pathophysiological factors. Anatol J Cardiol. 2018;20(5):275-282. doi:10.14744/AnatolJCardiol.2018.51447

11. KI Y, PARK K, KANG J, KIM C, HAN J, YANG H et al. Sa- fety and Efficacy of Second-Generation Drug-Eluting Stents in Real-World Practice: Insights from the Multicenter Grand-DES Registry. J Interv Cardiol. 2020;2020(1):1-9.

12. YANO H, HORINAKA S, WATAHIKI M, WATANABE T IT. Five-year outcomes after first- and second-generation drug-eluting stent implantation in all patients undergoing percutaneous coronary intervention. J Cardiol. 2019;74(1):169-174. doi:10.1016/j.jjcc.2019.01.012

13. ROSENBLUM J, HARSKAMP R HN, WALKER P, LIBERMAN H, DE WINTER R et al. Hybrid coronary revascularization versus coronary artery bypass surgery with bilateral or single internal mammary artery grafts. J Thorac Cardiovasc Surg. 2016;151(4):1081-1089.

14. GUAN Z, ZHANG Z, GU K, WANG H, LIN J, ZHOU W et al. Minimally invasive $\mathrm{CABG}$ or hybrid coronary revascularization for multivessel coronary diseases: Which is best? A systematic review and metaanalysis. Heart Surg Forum. 2019;23(1):494-502.

15. NARASIMHAN S, SRINIVAS V DJ. Hybrid coronary revascularization: A review. Cardiol Rev. 2011;19(3):101-107.

16. HALKOS M, WALKER P, VASSILIADES T, DOUGLAS J, DEVIREDDY C, GUYTON R et al. Clinical and angiographic results after hybrid coronary revascularization. Ann Thorac Surg. 2014;97(1):484-490.

17. HALKOS M, VASSILIADES T, DOUGLAS J, MORRIS D, RAB S, LIBERMAN H et al. Hybrid coronary revascularization versus off-pump coronary artery bypass grafting for the treatment of multivessel coronary artery disease. Ann Thorac Surg. 2011;92(1):1695-1701.

18. GASIOR M, ZEMBALA M, TAJSTRA M, FILIPIAK K, GIERLOTKA M, HRAPKOWICZ T et al. Hybrid revascularization for multivessel coronary artery disease. JACC Cardiovasc Interv. 2014;7(11):1277-1283.

19. SHEN L, HU S, WANG H, XIONG H ZZ, LI L et al. Onestop hybrid coronary revascularization versus coronary artery bypass grafting and percutaneous coronary intervention for the treatment of multivessel coronary artery disease: 3-Year follow-up results from a single institution. J Am Coll Cardiol. 2013;61(1):2525-2533. doi:10.1016/j.jacc.2013.04.007

20. GANYUKOV V, KOCHERGIN N, SHILOV A, TARASOV R, SKUPIEN J, SZOT W et al. Randomized Clinical Trial of Surgical vs. Percutaneous vs. Hybrid Revascularization in 
Multivessel Coronary Artery Disease: Residual Myocardial Ischemia and Clinical Outcomes at One Year - Hybrid coronary REvascularization Versus Stenting or Surgery (HREV. J Interv Cardiol. 2020;1(1):1-11.

21. SOUSA-UVA M, NEUMANN F, AHLSSON A, ALFONSO F, BANNING A, BENEDETTO U et al. 2018 ESC/EACTS Guidelines on myocardial revascularization. Eur Heart J. 2018;00(1):1-96.

22. SAYEDAHMAD Z, DARMOCH F, PACHA H M, S, MOHAMAD A YZ. HYBRID coronary revascularization versus coronary artery bypass graft in patients with multivessel coronary artery disease: a meta-analysis. J Am Coll Cardiol. 2020;75(11):1-2.

23. ZALAQUETT R, HOWARD M, MANUEL J IRARRÁZAVAL, MORAN S, MATURANA G, BECKER P, et al. Cirugía coronaria mínimamente invasiva. Rev Méd Chile 1999;127:45-52.
24. HARSKAMP R, BRENNAN J, XIAN Y, HALKOS M, PUSKAS J, THOURANI V et al. Practice patterns and clinical outcomes after hybrid coronary revascularization in the United States: an analysis from the society of thoracic surgeons adult cardiac database. Circulation. 2014;130(1):872-879.

25. LOWENSTERN A, WU J, BRADLEY S, FANAROFF A, TCHENG J, WANG T ET al. Current landscape of hybrid revascularization: A report from the NCDR CathPCI Registry. Am Heart J. 2019;215(1):167-177.

26. LAMY A, DEVEREAUX P, PRABHAKARAN D, TAGGART D, HU S, STRAKA Z et al. Five-year outcomes after off-pump or on-pump coronary-artery bypass grafting. N Engl J Med. 2016;375(1):2359-2368. doi:10.1056/NEJMoa1601564

27. PANOULAS V, COLOMBO A, MARGONATO A MF. Hybrid coronary revascularization: Promising, but yet to take off. J Am Coll Cardiol. 2015;65(1):85-97. doi:10.1016/j. jacc.2014.04.093. 